\title{
CONTRIBUCIÓN DE LOS LOCI Glu-B1, Glu-D1 Y Glu-B3 A LA CALIDAD DE LA MASA DEL TRIGO HARINERO
}

\section{CONTRIBUTION OF Glu-B1, Glu-D1 AND Glu-B3 LOCI TO DOUGH QUALITY OF BREAD WHEAT}

\author{
Eliel Martínez Cruz ${ }^{1}$, Eduardo Espitia Rangel ${ }^{\star 1}$, Héctor E. Villaseñor Mir ${ }^{1}$ y Roberto J. Peña Bautista ${ }^{2}$
} ${ }^{1}$ Programa de Trigo, Campo Experimental Valle de México, Instituto Nacional de Investigaciones Forestales, Agrícolas y Pecuarias. 56235, Chapingo, Estado de
México $^{2}$ Laboratorio de Calidad de Trigo, Centro Internacional de Mejoramiento de Maíz y Trigo. 56130, El Batán, Estado de México.

${ }^{*}$ Autor para correspondencia (espitia.eduardo@inifap.gob.mx)

\section{RESUMEN}

En esta investigación, se identificaron combinaciones y variantes alélicas de gluteninas de alto y bajo peso molecular (GAPM y GBPM), y se evaluó su efecto en las características reológicas de la masa de trigos harineros (Triticum aestivum L.). Se usaron los progenitores 'Bacanora T88' y 'Salamanca S75' y como progenie derivada de su cruza consistente en 98 líneas recombinantes obtenidas por descendencia de una sola semilla de $F_{2}$ a $F_{6}$. Los 100 genotipos fueron sembrados en Celaya, Guanajuato, México, durante el ciclo primavera-verano 2008. Las variables evaluadas fueron: tiempo de amasado, estabilidad al amasado, tolerancia al sobreamasado, fuerza general de la masa y la relación tenacidad/extensibilidad. Se identificaron las GAPM y GBPM en geles de poliacrilamida en presencia de dodecil sulfato de sodio. Se encontró que los loci Glu-B1, Glu-D1 y Glu-B3 afectaron las propiedades de fuerza y extensibilidad de la masa. La combinación de los alelos $2^{\star}, 7+9,5+10, c, g, b$ presentó gluten fuerte y excelente extensibilidad apto para la panificación, mientras que la combinación alélica $2^{\star}, 7+9,2+12, c, g, b$ condicionó gluten débil y extensible útil en la fabricación de galletas y tortillas. Las combinaciones $2^{\star}, 7+9,2+12 c, j, b$ y $2^{\star}, 7+8,2+12 c, j, b$ se asociaron con menor fuerza y mayor extensibilidad de la masa, respectivamente. Las variantes alélicas de GAPM 7+8, 7+9 y $5+10$ de los loci Glu-B1 y Glu-D1 se clasificaron como de gluten fuerte; mientras que $2+12$ se asoció con gluten medio fuerte. El alelo $j$ de GBPM del locus Glu-B3 presentó los valores más altos para la relación tenacidad/extensibilidad y los menores para fuerza, contrario a la variante $g$. Lo anterior muestra que mediante la selección de progenitores y la recombinación de sus alelos se pueden obtener genotipos de trigo harinero con calidad específica de la masa.

Palabras clave: Triticum aestivum, fuerza y extensibilidad de la masa, gluteninas de alto y bajo peso molecular.
SUMMARY

The aim of this research was to identify the combinations and allelic variants of high and low molecular weight glutenins (HMWG and LMWG) to determine their effects on the rheological characteristics of dough of bread wheat (Triticum aestivum $\mathrm{L}_{\text {.). }}$. A set of 98 lines derived by single seed descent from $F_{2}$ to $F_{6}$ of the cross 'Bacanora T88 x Salamanca' was used. Genotypes were sown at Celaya, Guanajuato, México, 2008 during the Winter-Spring cycle. Variables evaluated were:mixing time, mixing stability, tolerance to overmixing, dough strength and the tenacity/extensibility ratio. Both HMWG and LMWG were identified with sodium dodecyl sulfate in polyacrylamide gel. It was found that loci Glu-B1, Glu-D1 and Glu-B3 affected both dough strength and extensibility. The combination $2^{\star}, 7+9,5+10, c, g$, $b$ was associated to strong gluten and excellent extensibility, suitable for bread-making; in contrast, combination $2^{*}, 7+9,2+12, \mathrm{c}, \mathrm{g}, \mathrm{b}$ showed weak and extensible gluten, useful for manufacturing cookies and tortillas. The combination $2^{\star}, 7+9,2+12 c, j, b$ showed lowest dough strength and $2^{\star}, 7+8,2+12 c, j, b$ showed the highest extensibility. Alleles $7+9$ and $5+10$ were associated with higher gluten strength than alleles $7+8$ and $2+12$. The highest tenacity and lowest ratio tenacity/extensibility values were presented by the allele $j$, compared to $g$ allele. Therefore, by recombination of progenitors and selection of specific alleles it is possible to obtain bread wheat genotypes with specific quality of dough.

Index words: Triticum aestivum, dough strength and extensibility, high and low molecular weight glutenins.

\section{INTRODUCCIÓN}

La cohesividad, viscosidad y elasticidad de la masa del trigo harinero (Triticum aestivum L.) son propiedades reológicas que le confieren la fuerza y extensibilidad a la masa $y$, consecuentemente, determinan la calidad del producto final a obtener: panes, galletas, tortillas y pasteles 
(Wieser, 2007). Estas características reológicas (fuerza y extensibilidad) del gluten son controladas parcialmente por diversos alelos que codifican subunidades de proteínas denominadas gluteninas y gliadinas (Hoseney, 1991). Se ha demostrado que las gluteninas de alto peso molecular (GAPM), las gluteninas de bajo peso molecular (GBPM) y las gliadinas tienen efecto sobre la fuerza y extensibilidad de la masa (Liu et al., 2009; Zheng et al., 2009). En trigos mexicanos se han caracterizado a varios alelos de los GAPM e identificado sus efectos individuales sobre la reología de la masa (De la O et al., 2006; Martínez et al., 2007; Espitia et al., 2008), así como el efecto del ligamiento de $\omega$ (omega) con gliadinas/GBPM (Martínez et al., 2007). Además, se ha identificado la diversidad de combinaciones de las variantes alélicas de GAPM y GBPM, principalmente dentro del programa de mejoramiento para trigo de temporal (Martínez et al., 2010a); sin embargo, falta identificar el efecto individual de las GBPM y en combinación con las GAPM.

La traslocación 1BL.1RS involucra la transferencia de secalinas, proteínas del centeno (Secale cereale), al genoma del trigo harinero, mediante el remplazo del brazo corto del cromosoma 1B del trigo por el brazo corto $1 \mathrm{R}$ del centeno. Esta sustitución confiere al trigo resistencia a las royas de la hoja (Puccinia triticina) y amarilla ( $P$. striiformis) (Zeller y Hsam, 1983), además de aportar alto rendimiento y amplia adaptación (Kim et al., 2004). Sin embargo, dicha traslocación desfavorece la calidad del gluten (Liu et al., 2005), por lo que es necesario seleccionar genotipos con combinaciones de GAPM y GBPM que la demeriten en menor grado.

En México el trigo harinero se clasifica de acuerdo a la fuerza (W) y extensibilidad del gluten $(\mathrm{P} / \mathrm{L})$, en cuatro grupos: Grupo 1, gluten fuerte y extensible con $\mathrm{W}>300 \mathrm{x}$ $10^{-4} \mathrm{~J}$ y $\mathrm{P} / \mathrm{L}<1$; Grupo 2, gluten medio fuerte y extensible con W entre 200 y $300 \times 10^{-4} \mathrm{~J}$ y $\mathrm{P} / \mathrm{L}<1$; Grupo 3, gluten débil y extensible con $\mathrm{W}<200 \times 10^{-4} \mathrm{~J}$ y $\mathrm{P} / \mathrm{L}<1$; y Grupo 4, gluten tenaz poco extensible con valores de $\mathrm{P} / \mathrm{L}>1.2$ (Salazar et al., 2000).

De acuerdo con lo anterior, la fuerza y extensibilidad de la masa del trigo harinero es parcialmente definida por el efecto individual de GAPM y GBPM y el de sus combinaciones alélicas; esto obliga a explorar la gama de combinaciones de alelos en diversos progenitores y sus progenies para identificar las que consistentemente se asocien con características específicas de la calidad de la masa. Por ello que el objetivo de esta investigación fue identificar el efecto combinado e individual de las variantes alélicas de las GAPM y GBPM de líneas recombinantes derivadas de la cruza 'Bacanora T88 x Salamanca S75', sobre la fuerza y extensibilidad de la masa.

\section{MATERIALES Y MÉTODOS}

\section{Material genético}

Como progenitores se usaron a las variedades 'Bacanora T88' y 'Salamanca S75', y como progenie de tal cruza a 98 líneas $\mathrm{F}_{6}$ derivadas por descendencia de una sola semilla de $\mathrm{F}_{2}$ a $\mathrm{F}_{6}$. 'Bacanora T88' es una variedad de gluten débil (poca fuerza) y tenaz (Grupo 4), impropio para la panificación en forma mecanizada y semimecanizada (Martínez et al., 1989). La harina de 'Salamanca S75' produce masas débiles y balanceadas, ideales para la elaboracion de galletas, pasteles, tortillas inclusive y pan artesanal, ademas de ser útil en mezclas para corregir variedades que producen masas con fuerza excesiva (Solís, 2000).

Los 100 genotipos fueron sembrados durante el ciclo primavera-verano 2008 en el Campo Experimental Bajío en Roque, Guanajuato, conforme al paquete tecnológico recomendado por el Instituto Nacional de Investigaciones Forestales, Agrícolas y Pecuarias (INIFAP) para siembras de trigo de riego en esta zona (Solís, 2000), bajo un diseño experimental de bloques completos al azar con dos repeticiones. La unidad experimental consistió de cuatro surcos de $3 \mathrm{~m}$ de longitud y separados a $30 \mathrm{~cm}$.

La progenie se agrupó en cinco combinaciones (C) recombinantes generadas para identificar su efecto (Cuadro 1); mediante los contrastes entre las combinaciones $\mathrm{C} 1$ vs. $\mathrm{C} 2$ se identificaron los efectos individuales de las variantes alélicas $5+10$ y $2+12$ en GluD1, mediante C3 vs. C4 se identificó el efecto de las mismas variantes en presencia de la traslocación (alelo $j$ en Glu-B3). El efecto de $7+9$ y $7+8$ en Glu-B1, se identificó mediante los contrastes $\mathrm{C} 2$ vs. C5. Finalmente, mediante el contraste C4 vs. C5 se determinó el efecto de $j$ y $g$ en Glu-B3. Para el análisis estadístico se incluyeron las combinaciones de la progenie que no presentaron dos alelos en un locus, debido a que esto indica contaminación 
de la muestra con otra línea, lo que no permitiría identificar el efecto individual del alelo.

\section{Variables evaluadas en el laboratorio}

Los analisis de calidad reológica y la identificación de los genotipos con base en sus combinaciones GAPM y GBPM, se hicieron paralelamente en el Laboratorio de Calidad del Trigo del Centro Internacional de Mejoramiento de Maíz y Trigo.

Para la obtención de la harina refinada, a $500 \mathrm{~g}$ de grano se adicionó agua en una cantidad que dependió de la dureza y humedad del grano. La molienda se hizo en un molino Brabender Quadramat Senior ${ }^{\circledR}$ (C.W. Brabender OHG, Germany). La harina de la molienda fue cernida a través de una malla de diámetro $8 \mathrm{xx}$ y $10 \mathrm{xx}$, para la obtención de la harina refinada de prueba.

Las características mixográficas se determinaron en un mixógrafo de Swanson ${ }^{\circledR}$ (National Mfg., Lincoln, NE, USA.), conforme al método 54-40A (AACC, 2005). Este método consiste en amasar harina refinada con agua, la cual se adiciona con base en la humedad y la proteína de la muestra. El tiempo de desarrollo de la masa se detuvo a 3.5 min después del punto óptimo de amasado. Del mixograma generado se calculó: tiempo de amasado (TAM), estabilidad al amasado (EAM) y tolerancia al sobreamasado (TSAM). Valores altos en tiempo de amasado y estabilidad al amasado así como valores bajos en tolerancia al sobre amasado se asocian con mayor fuerza de la masa.

Para determinar las variables alveográficas se uso $50 \mathrm{~g}$ de harina refinada, y con base en la humedad se determinó la cantidad de solución agua-sal (2.5\%). Después del amasado se obtuvieron tres "láminas de masa" de un grosor constante, las cuales se dejaron en reposo durante $20 \mathrm{~min}$ a una temperatura de $25^{\circ} \mathrm{C}$. Posteriormente se sometieron a una presión constante de aire $y$ paralelamente se formó el globo de masa y el alveograma. De la gráfica generada (alveograma) se calculó la fuerza general de la masa (W) y la relación de tenacidad/extensibilidad (P/L). Dicho procedimiento se hizo en el alveógrafo de Chopin ${ }^{\circledR}$ (Trippette \& Renaud, Francie) con el método 54-30A (AACC, 2005).
La identificación de las variantes alélicas se hizo mediante el método electroforético descrito por Peña et al. (2004). Las GAPM (loci Glu-A1, Glu-B1 y Glu-D1) se identificaron con base en la nomenclatura propuesta por Payne y Lawrence (1983), y las GBPM (loci Glu-A3 y GluB3) de acuerdo con Singh et al. (1991), Jackson et al. (1996) y Branlard et al. (2003). Para la identificación de los alelos del locus Glu-D3 se usó la nomenclatura propuesta por Branlard et al. (2003). La translocación 1BL.1RS se identificó como el alelo $j$ en $G l u-B 3$, de acuerdo con Gupta y Shepherd, (1992).

\section{Análisis estadístico}

Se hizo un análisis de varianza general en cada variable estudiada. Además, a la fuente de variación genotipos se le aplicó un análisis de varianza adicional para obtener los cuadrados medios por combinación y contrastes ortogonales. También se hizo la comparación de medias mediante la prueba de DMS ( $\mathrm{P} \leq 0.05)$, con el procedimiento GLM de SAS (SAS Institute, 2002) para identificar las diferencias entre combinaciones.

\section{RESULTADOS Y DISCUSIÓN}

De las cinco combinaciones en las que se agruparon las líneas recombinantes derivadas de la cruza 'Bacanora T88 x Salamanca S75' (Cuadro 1), dos de ellas presentaron la traslocación (alelo $j$ en el locus Glu-B3), lo que permitió evaluar su comportamiento reológico asociado con diferentes variantes alélicas.

Se encontraron diferencias $(P \leq 0.05)$ entre genotipos y combinaciones en todas las variables reológicas de la masa evaluadas (Cuadro 2). En la comparacion entre las combinaciones 1 y 2 así como entre 4 y 5, se encontraron diferencias para todas las variables analizadas; en cambio, entre las combinaciones 3 y 4 no se encontraron diferencias para tolerancia al sobreamasado si en la relación tenacidad/extensibilidad. Entre las combinaciones 2 y 5 se encontraron diferencias para todas las variables medidas, excepto para la relacion tenacidad/extensibilidad. 
Cuadro 1. Combinaciones de gluteninas de alto peso molecular (GAPM) y gluteninas de bajo peso molecular (GBPM) de los progenitores y de las progenies analizadas.

\begin{tabular}{|c|c|c|c|c|c|c|c|}
\hline & \multicolumn{3}{|c|}{ GAPM } & \multicolumn{3}{|c|}{ GBPM } & \multirow[b]{2}{*}{$\mathrm{F}$} \\
\hline & Glu-A1 & Glu-B1 & Glu-D1 & Glu-A3 & Glu-B3 & Glu-D3 & \\
\hline \multicolumn{8}{|l|}{ Progenitores } \\
\hline 'Bacanora T88' & $2^{*}$ & $7+9$ & $5+10$ & $c$ & $j$ & $b$ & 3 \\
\hline 'Salamanca S75’ & $2^{*}$ & $7+8$ & $2+12$ & $c$ & $g$ & $b$ & 3 \\
\hline \multicolumn{8}{|c|}{ Combinaciones de la progenie } \\
\hline 1 & $2^{*}$ & $7+8$ & $2+12$ & $c$ & $g$ & $b$ & 15 \\
\hline 2 & $2^{*}$ & $7+8$ & $5+10$ & $c$ & $g$ & $b$ & 15 \\
\hline 3 & $2^{*}$ & $7+9$ & $2+12$ & $c$ & $j$ & $b$ & 15 \\
\hline 4 & $2^{*}$ & $7+9$ & $5+10$ & $c$ & $j$ & $b$ & 15 \\
\hline 5 & $2^{*}$ & $7+9$ & $5+10$ & $c$ & $g$ & $b$ & 15 \\
\hline
\end{tabular}

$\mathrm{F}=$ frecuencia de los progenitores y la progenie.

Cuadro 2. Cuadrados medios de variables reologicas de la masa de trigos harineros.

\begin{tabular}{|c|c|c|c|c|c|c|}
\hline FV & $\mathrm{gl}$ & TAM & EAM & TSAM & $\mathrm{W}$ & $\mathrm{P} / \mathrm{L}$ \\
\hline Genotipo & 26 & $1.1^{\star *}$ & $3.4^{\star \star}$ & $45.9^{\star *}$ & $32617.0^{\star *}$ & $0.7^{* *}$ \\
\hline Combinación (C) & 6 & $3.7^{* *}$ & $12.1^{* *}$ & $152.8^{* *}$ & $97147.6^{* *}$ & $1.7^{\star *}$ \\
\hline $\mathrm{C} 1$ vs. $\mathrm{C} 2$ & 1 & $3.4^{* *}$ & $13.4^{* *}$ & $100.8^{* *}$ & $67497.6^{* *}$ & $1.7^{* *}$ \\
\hline C3 vs. C4 & 1 & $1.9^{* *}$ & $1.3^{*}$ & 14.7 & $18204.0^{*}$ & 0.07 \\
\hline C2 vs. C5 & 1 & 0.2 & 0.3 & 0.13 & 6541.6 & $1.3^{\star *}$ \\
\hline $\mathrm{C} 4 v s . \mathrm{C} 5$ & 1 & $4.7^{\star \star}$ & $30.2^{* *}$ & $360.5^{\star \star}$ & $227418.1^{\star *}$ & $2.3^{* *}$ \\
\hline Error & 48 & 0.02 & 0.08 & 2.6 & 637.8 & 0.04 \\
\hline $\mathrm{CV}(\%)$ & & 5.9 & 10.6 & 12 & 8.3 & 18.1 \\
\hline
\end{tabular}

$\mathrm{FV}$ = fuente de variación; $\mathrm{gl}=$ grados de libertad; $\mathrm{CV}=$ coeficiente de variación; ${ }^{9}=$ error del análisis general, VSED = Volumen de sedimentación, ISED = índice de sedimentación; TAM = tiempo de amasado; EAM = estabilidad al amasado; TSAM = tolerancia al sobreamasado; $\mathrm{W}=$ fuerza de la masa; $\mathrm{P} / \mathrm{L}=$ relacion tenacidad/extensibilidad.

${ }^{*}=$ significativas con $\mathrm{P} \leq 0.05$

$* *$ significativas con $\mathrm{P} \leq 0.01$

\section{Efecto por combinacion}

La combinación $1,7+9,5+10, c, j, b$ de 'Bacanora T88' y de las líneas recombinantes, mostró tiempos cortos de amasado y estabilidad (Cuadro 3). Para la tolerancia al sobreamasado, la combinacion $1,7+9,2+12, c, j, b$ se asoció con valores altos, lo que indica menor fuerza. Las combinaciones $2^{*}, 7+8,5+10, c, g, b$ y las similares a 'Salamanca S75', se asociaron con valores más altos de tiempo de amasado, estabilidad al amasado y valores bajos de tolerancia al sobreamasado, características que se asocian con mayor fuerza de gluten, de acuerdo con lo reportado por Martín et al. (2001) y Martínez et al. (2010b).
Las combinaciones recombinantes con GAPM $2^{*}, 7+9$ ó $7+8,5+10$, asociadas con las GBPM $c, g$ y $b$, fueron las que mostraron valores mayores a $300 \times 10^{-4} \mathrm{~J}$ clasificados como de gluten fuerte apto para la industria mecanizada o para usarse en mezclas para corregir masas débiles (Cuadro 4). La combinación $2^{*}, 7+9,5+10, c, g, b$ se asoció con excelente extensibilidad de la masa. Las combinaciones de GAPM $2^{*}, 7+9,5+10$ ó $2^{\prime}+12$ asociadas con las GBPM $c, j, b$, mostraron los valores más bajos de fuerza, clasificados como masas suaves o débiles; además, presentaron valores iguales o mayores a la unidad en la relación tenacidad/extensibilidad, propios de masas tenaces, lo que demerita la calidad reológica de la masa. Lo anterior concuerda con lo reportado por Li et al. (2010) quienes encontraron que la presencia del alelo $2+12$ y la 
traslocación reducen considerablemente la fuerza del gluten. Las combinaciones que presentaron los alelos $j$ y $b$ de los loci Glu-B3 y Glu-D3, respectivamente, redujeron la fuerza de la masa, como reportaron Gobaa et al. (2008). Las GBPM $c, g y b$ de las líneas recombinantes se asociaron con mayor extensibilidad de la masa, y la combinación $2^{*}$, $7+8,2+12, c, g, b$ fue la de mayor extensibilidad estas características son adecuadas para la elaboración de productos de la industria galletera y para la fabricación de tortilla. Consistentemente, las combinaciones recombinantes se asociaron con masas de mayor extensibilidad respecto a los progenitores, lo cual puede deberse a la presencia de gliadinas, no identificadas en este estudio, que favorecen esta característica como demostraron Khatkar et al.(2002).

Cuadro 3. Efecto de las combinaciones de gluteninas de alto y bajo peso molecular (GAPM y GBPM) en líneas recombinantes de trigo y en progenitores 'Bacanora T88' y 'Salamanca S75', en las variables mixográficas.

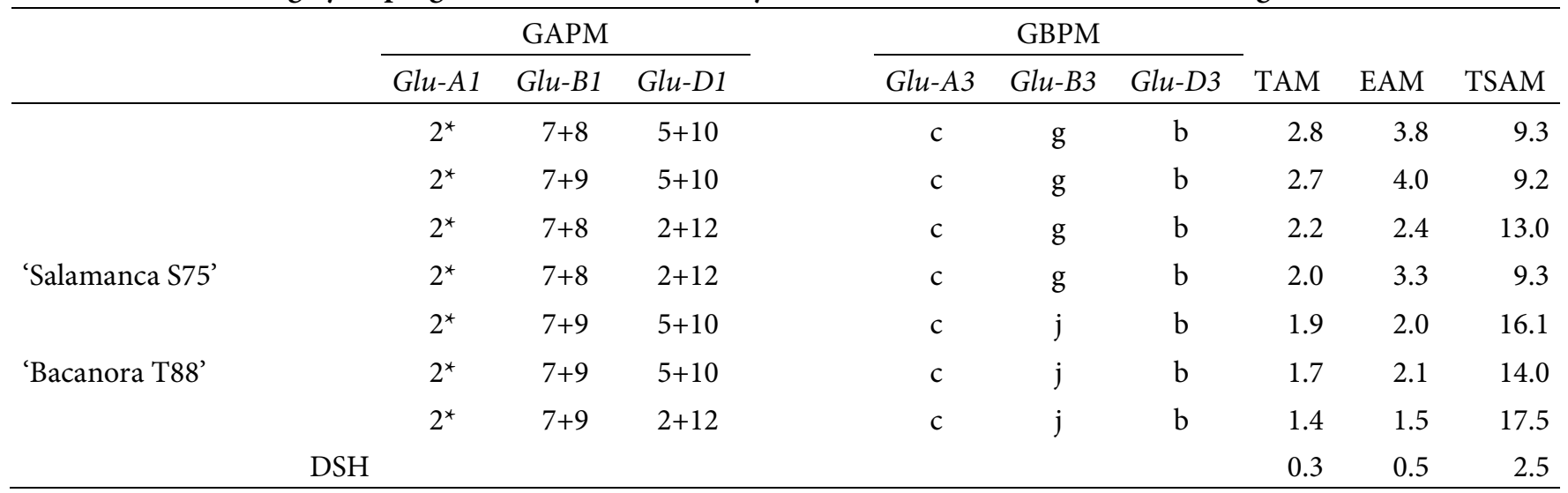

Variables mixográficas: tiempo de amasado (TAM, min), estabilidad al amasado (EAM, min), y tolerancia al sobreamasado $(\mathrm{TSAM}, \mathrm{mm}) . \mathrm{DSH}=$ diferencia significativa honesta.

Cuadro 4. Efecto de las combinaciones de gluteninas de alto y bajo peso molecular (GAPM y GBPM) en líneas recombinantes de trigo y en los progenitores 'Bacanora T88' y 'Salamanca S75', sobre las variables alveográficas.

\begin{tabular}{|c|c|c|c|c|c|c|c|c|c|}
\hline & \multicolumn{3}{|c|}{ GAPM } & \multicolumn{3}{|c|}{ GBPM } & \multirow[b]{2}{*}{$\mathrm{W}$} & \multirow[b]{2}{*}{$\mathrm{P} / \mathrm{L}$} \\
\hline & & Glu-A1 & Glu-B1 & Glu-D1 & Glu-A3 & Glu-B3 & Glu-D3 & & \\
\hline \multirow{5}{*}{ 'Salamanca S75' } & & $2^{*}$ & $7+9$ & $5+10$ & c & g & $\mathrm{b}$ & 411.3 & 0.8 \\
\hline & & $2^{*}$ & $7+8$ & $5+10$ & c & g & $\mathrm{b}$ & 381.7 & 1.2 \\
\hline & & $2^{*}$ & $7+8$ & $2+12$ & c & g & $\mathrm{b}$ & 330.7 & 1.6 \\
\hline & & $2^{*}$ & $7+8$ & $2+12$ & c & g & $\mathrm{b}$ & 286.9 & 0.7 \\
\hline & & $2^{*}$ & $7+9$ & $5+10$ & c & j & $\mathrm{b}$ & 237.1 & 1.3 \\
\hline \multirow{3}{*}{ ‘Bacanora T88’ } & & $2^{*}$ & $7+9$ & $2+12$ & c & j & $\mathrm{b}$ & 187.9 & 1.4 \\
\hline & & $2^{*}$ & $7+9$ & $5+10$ & c & j & $\mathrm{b}$ & 178.7 & 2.1 \\
\hline & DSH & & & & & & & 68.8 & 0.4 \\
\hline
\end{tabular}

Variables alveograficas: fuerza de la masa $\left(\mathrm{W}, 10^{-4} \mathrm{~J}\right)$, y relación tenacidad/extensibilidad (P/L, 0.1 a 6.0 ). DSH = diferencia significativa honesta. 
Cuadro 5. Comparación de medias de propiedades reológicas de la masa de alelos de Gluteninas de Alto Peso Molecular (GAPM) en líneas de trigo derivadas de la cruza 'Bacanora T88' y 'Salamanca S75'.

\begin{tabular}{lcccccc}
\hline \multirow{2}{*}{ Locus } & \multicolumn{2}{c}{ Glu-B1 } & & \multicolumn{2}{c}{ Glu-D1 } \\
\cline { 2 - 3 } \cline { 5 - 6 } Alelo/Variable & $7+9$ & $7+8$ & & & $5+10$ & $2+12$ \\
\hline Tiempo de amasado (min) & $2.7 \mathrm{a}$ & $2.8 \mathrm{a}$ & & $2.8 \mathrm{a}$ & $2.2 \mathrm{~b}$ \\
Estabilidad al amasado (min) & $4.0 \mathrm{a}$ & $3.8 \mathrm{a}$ & & $3.8 \mathrm{a}$ & $2.4 \mathrm{~b}$ \\
Tolerancia al sobreamasado $(\mathrm{mm})$ & $9.2 \mathrm{a}$ & $9.3 \mathrm{a}$ & & $9.3 \mathrm{a}$ & $13.0 \mathrm{~b}$ \\
Fuerza de la masa $\left(\mathrm{x} 10^{-4} \mathrm{~J}\right)$ & $411.3 \mathrm{a}$ & $381.7 \mathrm{a}$ & & $381.7 \mathrm{a}$ & $286.9 \mathrm{~b}$ \\
Tenacidad/extensibilidad $(0.1-6.0)$ & $0.8 \mathrm{a}$ & $1.2 \mathrm{~b}$ & & 1.2 & $0.7 \mathrm{~b}$ \\
\hline
\end{tabular}

Valores con la misma letra en una hilera son estadistícamente iguales (DMS, 0.05).

Cuadro 6. Comparación de medias de propiedades reológicas de la masa de alelos de Gluteninas de Bajo Peso Molecular (GBPM) en líneas de trigo derivadas de la cruza 'Bacanora T88' y 'Salamanca S75'.

\begin{tabular}{lcc}
\hline Locus & \multicolumn{2}{c}{ Glu-B3 } \\
\cline { 2 - 3 } Alelo/Variable & $1.9 \mathrm{a}$ & $2.7 \mathrm{~b}$ \\
\hline Tiempo de amasado $(\mathrm{min})$ & $2.0 \mathrm{a}$ & $4.0 \mathrm{~b}$ \\
Estabilidad al amasado $(\mathrm{min})$ & $16.1 \mathrm{a}$ & $9.2 \mathrm{~b}$ \\
Tolerancia al sobreamasado $(\mathrm{mm})$ & $237.1 \mathrm{a}$ & $411.3 \mathrm{~b}$ \\
Fuerza de la masa $\left(10^{-4} \mathrm{~J}\right)$ & $1.3 \mathrm{a}$ & $0.8 \mathrm{~b}$ \\
Tenacidad/extensibilidad $(0.1-6.0)$ &
\end{tabular}

Valores con la misma letra en una hilera son estadistícamente iguales (DMS, 0.05).

Cuadro 7. Comparación de medias de propiedades reológicas de la masa de alelos de Gluteninas de Alto Peso Molecular (GAPM) en presencia de 1BL.1RS, en líneas derivadas de la cruza 'Bacanora T88' y 'Salamanca S75'.

\begin{tabular}{lcc}
\hline Locus & \multicolumn{2}{c}{ Glu-D1 } \\
\cline { 2 - 3 } Alelo/Variable & $5+10$ & $2+12$ \\
\hline Tiempo de amasado $(\mathrm{min})$ & $1.9 \mathrm{a}$ & $1.3 \mathrm{~b}$ \\
Estabilidad al amasado $(\mathrm{min})$ & $2.0 \mathrm{a}$ & $1.5 \mathrm{a}$ \\
Tolerancia al sobreamasado $(\mathrm{mm})$ & $16.1 \mathrm{a}$ & $17.5 \mathrm{a}$ \\
Fuerza de la masa $\left(10^{-4} \mathrm{~J}\right)$ & $237.1 \mathrm{a}$ & $187.9 \mathrm{a}$ \\
Tenacidad/extensibilidad $(0.1-6.0)$ & $1.3 \mathrm{a}$ & $1.4 \mathrm{a}$ \\
\hline
\end{tabular}

Valores con la misma letra en una hilera son estadistícamente iguales (DMS, 0.05).

\section{Efecto por alelo}

La comparación de medias de los genotipos agrupados por los alelos contrastantes en los loci Glu-B1 y Glu-D1 que codifican para GAPM, se muestran en Cuadro 5. Los grupos representados por los alelos $7+8$ y $7+9$ se asociaron con características similares de amasado. Por la fuerza de sus masas se agruparon como fuertes, pero su relación de tenacidad/extensibilidad $7+9$ favoreció la extensibilidad y $7+8$ se asoció con masas balanceadas. En el caso de $5+10$ vs. $2+12$, de manera general $5+10$ presentó tiempos mayores de amasado y de estabilidad así como valores bajos de tolerancia al sobreamasado lo cual significa mayor fuerza, en concordancia con Yanaka et al. (2007) y Li et al. (2010), quienes asociaron a este alelo con alta fuerza de la masa. Por su parte, $2+12$ se clasificó como gluten medio fuerte con excelente extensibilidad.

Los genotipos agrupados por los alelos contrastantes en el locus Glu-B3, que codifican para GBPM, se muestran en Cuadro 6. La variante alélica $g$ presentó 
valores característicos de gluten fuerte $\left(>300 \times 10^{-4} \mathrm{~J}\right)$ con excelente extensibilidad, por su relación tenacidad/extensibilidad, < 1.0; resultados iguales fueron encontrados por Meng et al. (2007) y Peña et al. (2004). En cambio, que el alelo $j$ se asoció con masas medio fuertes pero de alta tenacidad, por su valor de la relación tenacidad/extensibilidad $>1.2$ lo cual se asocia con masas de menor calidad reológica, según reportado por Meng et al. (2007) y Li et al. (2010).

En el Cuadro 7 se presenta la comparación de medias de los alelos $5+10$ y $2+12$ con la presencia de la traslocación, alelo $j$ en Glu-B3. En ambas variantes, se observó que la presencia de la traslocación demeritó la fuerza y favoreció la tenacidad de la masa representada por su relación tenacidad/extensibilidad $>1.2$ lo cual concuerda con lo reportado por Liu et al. (2005).

Otro factor que afecta la calidad reológica de la masa es la cantidad de proteína en la harina refinada, pero entre los contrastes de las combinaciones comparadas en la presente investigación no hubo diferencias significativas (datos no presentados).

\section{CONCLUSIONES}

Los loci Glu-B1, Glu-D1 y Glu-B3 influyen en las propiedades reológicas de la masa. La combinación recombinante $2^{*}, 7+9,5+10, c, g, b$ se asoció con valores de gluten fuerte y excelente extensibilidad, apto para la industria de la panificación mecanizada. En contraste, la combinación $2^{*}, 7+9,2+12, c, g, b$ de gluten débil $y$ extensible presentó características adecuadas para la fabricación de galletas y tortillas.

Las combinaciones recombinantes $2^{*}, 7+9,2+12 c, j$, $b$ y $2^{*}, 7+8,2+12 c, j, b$ se asociaron con los valores menores de fuerza y extensibilidad de la masa, respectivamente. Las variantes alélicas de GAPM $7+8$, $7+9$ y $5+10$ de los loci Glu-B1 y Glu-D1 se clasificaron como de gluten fuerte.

El alelo $j$ de GBPM del locus Glu-B3 presentó los valores más altos de la relación tenacidad/extensibilidad y menores para fuerza, contrario a la variante $g$. Lo anterior indica que mediante la recombinación y selección, con base en los alelos y las combinaciones de
GAPM y GBPM, se pueden generar genotipos de trigo con calidad específica de la masa.

\section{BIBLIOGRAFÍA}

AACC, American Association of Cereal Chemists (2005) Approved Methods of the AACC. 10th ed. American Association of Cereal Chemists. St. Paul, MN, USA.

Branlard G, M Dardevet, N Amiour, G Igrejas (2003) Allelic diversity of HMW and LMW glutenin subunits and omegagliadins in French bread wheat (Triticum aestivum L.). Genet. Resour. Crop Evol. 50: 669-679.

De la O O M, E Espitia R, J D Molina G, R J Peña B, A Santacruz V, H E Villaseñor M (2006) Efecto de diferentes subunidades de gluteninas-APM sobre la calidad panadera en trigos harineros mexicanos. Rev. Fitotec. Mex. 29: 291-297.

Espitia R E, E Martínez C, R J Peña B, H E Villaseñor M, J Huerta E (2008) Polimorfismo de gluteninas de alto peso molecular $y$ su relación con trigos harineros para temporal. Agric. Téc. Méx. 34: 57-67.

Gobaa S, C Brabant, G Kleijer, P Stamp (2008) Effect of the 1BL.1RS translocation and of the Glu-B3 variation on fifteen quality tests in a doubled haploid population of wheat (Triticum aestivum L.). J. Cereal Sci. 48:598-603.

Gupta R B, K W Shepherd (1992) Identification of rye chromosome $1 \mathrm{R}$ translocations and subunits in hexaploid wheats using storage proteins as genetic markers. Plant Breed. 109:130-140.

Hoseney R C (1991) Principios de Ciencia y Tecnología de Cereales. Ed. Acribia. Zaragoza, España. 321 p.

Jackson E A, M H Morel, T Sontag-Strohm, G Branlard, E V Metakovsky, R Redaelli (1996) Proposal for combining the classification systems of alleles of Gli-1 and Glu-3 loci in bread wheat (Triticum aestivum L.). J. Genet. and Breed. 50:321-336.

Khatkar S B, R J Fido, A S Tatham, J D Schofield (2002) Functional properties of wheat gliadins. I. Effects on mixing characteristics and bread making quality. J. Cereal Sci. 35:299306.

Kim W, J W Johnson, P S Baenziger, A J Lukaszewski, C S Gaines (2004) Agronomic effect of wheat-rye translocation carrying rye chromatin (1R) from different sources. Crop Sci. 44: 12541258.

Li Y, R Zhou, G Branlard, J Jia (2010) Development of introgression lines with 18 alleles of glutenin subunits and evaluation of the effects of various alleles on quality related traits in wheat (Triticum aestivum L.). J. Cereal Sci. 51:127-133.

Liu L, Z H He, Y Yan, X C Xia, R J Peña (2005) Allelic variations at the Glu-1 and Glu-3 loci, presence of the 1B.1R translocation, and their effects on mixographic properties in Chinese bread wheats. Euphytica 142:197-204.

Liu L, Z H He, W J Ma, J J Liu, X C Xia, R J Peña (2009) Allelic variation at the Glu-D3 locus in Chinese bread wheat and effects on dough properties, pan bread and Noodle qualities. Cereal Res. Comm. 37:57-64.

Martín P, M Gómez, J M Carrillo (2001) Interaction between allelic variation at the Glu-D1 locus and 1BL.1RS translocation on flour quality in bread wheat. Crop Sci. 41:1080-1084.

Martínez C E, E Espitia R, I Benítez R, R J Peña B, A Santacruz V, H E Villaseñor M (2007) El complejo GLI-1/GLU-3 y las 
propiedades reológicas y volumen de pan de trigos harineros. Rev. Fitotec. Mex. 30:167-172.

Martínez C E, E Espitia R, H E Villaseñor M, J D Molina G, I Benítez R, A Santacruz V, R J Peña B (2010a) Diversidad genética de gluteninas y gliadinas en trigos harineros (Triticum aestivum L.) mexicanos. Agrociencia 44:187-195.

Martínez C E, E Espitia R, H E Villaseñor M, J D Molina G, I Benítez R, A Santacruz V, R J Peña B (2010b) Diferencias reológicas de la masa de trigo en líneas recombinantes. II. Relación con combinaciones de los loci Glu-1 y Glu-3. Agrociencia 44:631-641.

Martínez S J de J, P F Valencia, P F López (1989) Cumpas T88 y Bacanora T88: nuevas variedades de trigo harinero. Folleto Técnico No. 12. SARH-INIFAP. México. $16 \mathrm{p}$.

Meng X G, F Xie, X W Shang, L Z An (2007) Association between allelic variations at the Glu-3 loci and wheat quality traits with lanzhou alkaline stretched noodles quality in northwest China spring wheats. Cereal Res. Comm. 35:109-118.

Payne P I, G J Lawrence (1983) Catalogue of alleles for the complex loci Glu-A1, Glu-B1 and Glu-D1, which code for highmolecular-weight subunits of glutenin in hexaploid wheat. Cereal Res. Comm. 11:29-35.

Peña B R J, H González S, F Cervantes (2004) Relationship between Glu-D1/Glu-B3 allelic combinations and breadmaking quality-related parameters commonly used in wheat breeding. In: The Gluten Proteins. D Lafiandra, S Masci, R D'Ovidio (eds). RSC Publishing, Cambridge. UK. pp:156-157.
Solís M E (2000) Variedades y época de siembra. In: Trigo de Riego Origen, Variedades, Manejo de Cultivo, Calidad Industrial. E Solís, A Rodríguez (eds). SAGAR, INIFAP. México. pp:25-31.

Salazar Z A (2000) Calidad industrial del trigo para su comercialización. In: El Trigo de Temporal en México. H E Villaseñor, E Espitia (eds). SAGAR, INIFAP. México. pp:192207.

SAS Institute (2002) SAS/STAT User's Guide, Software version 9.0. Cary, N. C, USA. 4424.

Singh N K, K W Shepherd, G B Cornish (1991) A simplified SDSPAGE procedure for separating LMW subunits of glutenin. J. Cereal Sci. 14:203-208.

Wieser H (2007) Chemistry of gluten proteins. Food Microbiol. 24:115-119.

Yanaka M, K Takata, T M Ikeda, N Ishikawa (2007) Effect of the high molecular weigh glutenin allele, Glu-d1d, on Noodle quality of common wheat. Breed. Sci. 57:243-248.

Zeller F J, S L K Hsam (1983) Broadening the genetic variability of cultivated wheat by utilizing rye chromatin. In: Proc. of the 6th International Wheat Genetics Symposium. S Sakamoto (ed). Kyoto University, Kyoto. pp:161-173.

Zheng S, P F Byrne, G Bai, X Shan, S D Reid, S D Haley, B W Seabourn (2009) Association analysis reveals effects of wheat glutenin alleles and rye translocations on dough-mixing properties. J. Cereal Sci. 50:283-290. 